\title{
Genome-wide identification and analysis of the thiolase family in insects
}

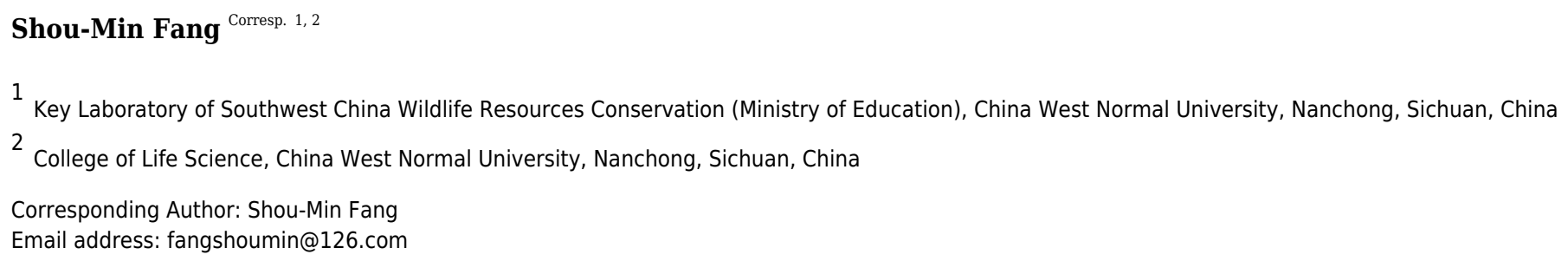

Thiolases are important enzymes involved in lipid metabolism in both prokaryotes and eukaryotes, and are essential for a range of metabolic pathways, while, little is known for this important family in insects. To shed light on the evolutionary models and functional diversities of the thiolase family, 137 thiolase genes were identified in 20 representative insect genomes. They were mainly classified into five classes, namely cytosolic thiolase (CT-thiolase), T1-thiolase, T2-thiolase, trifunctional enzyme thiolase (TFE-thiolase), and sterol carrier protein 2 thiolase (SCP2-thiolase). The intron number and exon/intron structures of the thiolase genes reserve large diversification. Subcellular localization prediction indicated that all the thiolase proteins were mitochondrial, cytosolic, or peroxisomal enzymes. Four highly conserved sequence fingerprints were found in the insect thiolase proteins, including CxS-, NEAF-, GHP-, and CxGGGxG-motifs. Homology modeling indicated that insect thiolases share similar 3D structures with mammals, fishes, and microorganisms. In Bombyx mori, microarray data and reverse transcriptionpolymerase chain reaction (RT-PCR) analysis suggested that some thiolases might be involved in steroid metabolism, juvenile hormone $(\mathrm{JH})$, and sex pheromone biosynthesis pathways. In general, sequence and structural characteristics were relatively conserved among insects, bacteria and vertebrates, while different classes of thiolases might have differentiation in specific functions and physiological processes. These results will provide an important foundation for future functional validation of insect thiolases. 


\section{Genome-wide identification and analysis of the}

2 thiolase family in insects

3

4

5

6

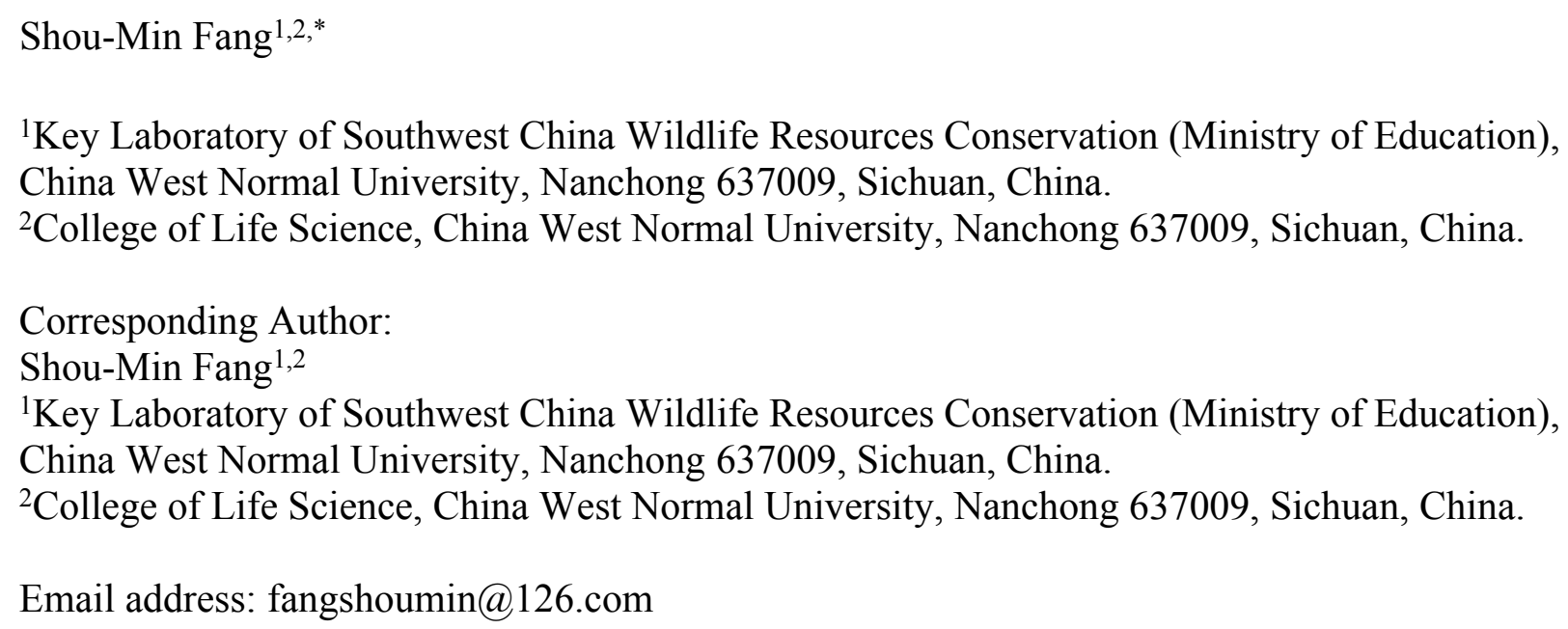

${ }^{1}$ Key Laboratory of Southwest China Wildlife Resources Conservation (Ministry of Education), China West Normal University, Nanchong 637009, Sichuan, China.

${ }^{2}$ College of Life Science, China West Normal University, Nanchong 637009, Sichuan, China.

Corresponding Author:

Shou-Min Fang ${ }^{1,2}$

${ }^{1}$ Key Laboratory of Southwest China Wildlife Resources Conservation (Ministry of Education), China West Normal University, Nanchong 637009, Sichuan, China.

${ }^{2}$ College of Life Science, China West Normal University, Nanchong 637009, Sichuan, China.

Email address: fangshoumin@126.com

\section{Abstract}

Thiolases are important enzymes involved in lipid metabolism in both prokaryotes and eukaryotes, and are essential for a range of metabolic pathways, while, little is known for this important family in insects. To shed light on the evolutionary models and functional diversities of the thiolase family, 137 thiolase genes were identified in 20 representative insect genomes. They were mainly classified into five classes, namely cytosolic thiolase (CT-thiolase), T1-thiolase, T2-thiolase, trifunctional enzyme thiolase (TFE-thiolase), and sterol carrier protein 2 thiolase (SCP2-thiolase). The intron number and exon/intron structures of the thiolase genes reserve large diversification. Subcellular localization prediction indicated that all the thiolase proteins were mitochondrial, cytosolic, or peroxisomal enzymes. Four highly conserved sequence fingerprints were found in the insect thiolase proteins, including CxS-, NEAF-, GHP-, and CxGGGxG-motifs. Homology modeling indicated that insect thiolases share similar 3D structures with mammals, fishes, and microorganisms. In Bombyx mori, microarray data and reverse transcription-polymerase chain reaction (RT-PCR) analysis suggested that some thiolases might be involved in steroid metabolism, juvenile hormone $(\mathrm{JH})$, and sex pheromone biosynthesis pathways. In general, sequence and structural characteristics were relatively conserved among insects, bacteria and vertebrates, while different classes of thiolases might have differentiation in specific functions and physiological processes. These results will provide an important foundation for future functional validation of insect thiolases.

\section{Introduction}


39 Thiolases are ubiquitous enzymes that play important roles in lipid-metabolizing pathways 40 (Thompson et al. 1989; Igual et al. 1992; Pereto, Lopez-Garcia \& Moreira 2005). There are two 41 major kinds of thiolases based on the direction of the catalytic reaction (Masamune et al. 1989; 42 Modis \& Wierenga 2000). One is degradative thiolase I (3-ketoacyl-CoA thiolase, E.C. 2.3.1.16),

which catalyzes the thiolytic cleavage of medium- to long-chain unbranched 3-oxoacyl-CoAs (from 4 to 22 carbons) into acetyl-CoA and a fatty acyl-CoA (Fig. S1A) (Clinkenbeard et al. 1973, Schiedl et al. 2004, Houten \& Wanders 2010). It is mainly involved in fatty acid $\beta$-oxidation and preferentially catalyzes the last step. The other is biosynthetic thiolase II (acetoacetyl-CoA thiolase, EC2.3.1.9), which is capable of catalyzing the Claisen condensation reaction of two molecules of acetyl-CoA to acetoacetyl-CoA (Fig. S1B). Thiolase II might be involved in poly beta-hydroxybutyric acid synthesis, steroid biogenesis, etc. (Clinkenbeard et al. 1973).

Based on the function, oligomeric state, substrate specificity, and subcellular localization, six different classes of thiolases (CT, AB, SCP2, T2, T1, and TFE) have been identified in humans (Fukao 2002; Mazet et al. 2011; Anbazhagan et al. 2014). In trypanosomatid and bacterial kingdoms, another four classes were identified, including thiolase-like protein (TLP), SCP2thiolase-like protein (SLP), unclassified thiolase (UCT), and TFE-like thiolase (TFEL) (Mazet et al. 2011; Anbazhagan et al. 2014). The CT-thiolase, located in the cytosol, has a key role in catalyzing the condensation of two molecules of acetyl-CoA to acetoacetyl-CoA, which is the first reaction of the metabolic pathway leading to the synthesis of cholesterol (Kursula et al. 2005). AB-thiolase and SCP2-thiolase as degradative thiolases occur in peroxisomes (Antonenkov et al. 1997; Antonenkov et al. 1999). T1-, T2-, and TFE-thiolases are mitochondrial degradative enzymes. Except for the degradation of acetoacetyl-CoA and 2-methyl-acetoacetyl-CoA, T2thiolase has a biosynthetic function in the synthesis of acetoacetyl-CoA in ketone body metabolism 
62 (Fukao et al. 1997). In general, AB-, SCP2-, T1-, and TFE-thiolases belong to degradative thiolase

63 I, and CT class is biosynthetic thiolase II. Importantly, T2-thiolase is a bi-functional enzyme with 64 synthesis and degradation activity.

65 As enzymes responsible for broad pathways, thiolases have been performed the 66 phylogenetic analysis in mycobacteria and functional studies in humans (Mazet et al. 2011; Xia et 67 al. 2019). In insects, several thiolase genes have been characterized (Fujii et al. 2010). In 68 Helicoverpa armigera, an acetoacetyl-CoA thiolase was cloned and performed functional analysis 69 (Zhang et al. 2017). It was indicated that the thiolase was involved in the early step of the juvenile 70 hormone pathway, i.e. mevalonate biosynthesis. One acetoacetyl-CoA thiolase was purified to 71 apparent homogeneity by column chromatography in Bombus terrestris, suggesting that it might 72 be the first enzyme in the biosynthesis of terpenic sex pheromone (Brabcova et al. 2015). Besides, 73 acetyl-CoA can be used as the precursor for de novo biosynthesis of sex pheromones in female 74 moths, and four 3-ketoacyl-CoA thiolase genes were identified in sex pheromone gland 75 transcriptome of the noctuid moth Heliothis virescens (Vogel et al. 2010). In total, there are few 76 and scattered studies on insect thiolase, lacking systematic identification and comparative studies. 77 In this study, we selected 20 representative species from 7 insect orders to perform genome78 wide identification of the thiolase family proteins. Gene structure, chromosome location, and 79 three-dimensional (3D) structure and motif characteristics of proteins were compared. In addition, 80 Bombyx mori is an important model species for studying juvenile hormone and sex pheromone 81 biosynthesis (Matsumoto 2010; Xia, Li \& Feng 2014). Expression profiles of the thiolase genes 82 were detected in various tissues and developmental sex pheromone gland of B. mori. Combining 83 structural characteristics and expression patterns, the potential functions and involved 
84 physiological processes were hypothesized. The present study can help us understand the

85 functional differentiation of thiolase genes in insects.

86 Materials \& Methods

87 Data resources

88 In this study, 20 representative species were selected from Lepidoptera, Hymenoptera, Hemiptera, 89 Diptera, Coleoptera, Phthiraptera, and Isoptera (Table 1). The annotated genes and genomes of $B$. 90 mori were retrieved from SilkDB v3.0 (https://silkdb.bioinfotoolkits.net). The sequence 91 information of Danaus plexippus and Heliconius melpomene were downloaded from 92 http://metazoa.ensembl.org/. Manduca sexta was from $93 \mathrm{ftp}: / / \mathrm{ftp}$. bioinformatics.ksu.edu/pub/Manduca/OGS2/. The other sequences were retrieved from 94 GenBank (https://www.ncbi.nlm.nih.gov/), including Papilio xuthus, Plutella xylostella, Culex 95 quinquefasciatus, Anopheles gambiae, Drosophila melanogaster, Anoplophora glabripennis, 96 Nicrophorus vespilloides, Tribolium castaneum, Halyomorpha halys, Acyrthosiphon pisum, Cimex 97 lectularius, Diachasma alloeum, Apis mellifera, Bombus impatiens, Pediculus humanus, and 98 Zootermopsis nevadensis.

\section{Identification of insect thiolase genes}

100 The known thiolase sequences of Homo sapiens and Mycobacterium tuberculosis were retrieved 101 from GenBank (Table S1; Anbazhagan et al. 2014) and used as queries to perform BLASTP search 102 (E-value $<0.01)$ against the protein database of predicted genes in each species. Hidden Markov 103 Model (HMM) files of Thionlase_N (PF00108) and Thionlase_C (PF02803) domains were 104 downloaded from Pfam database (http://pfam.xfam.org/), which were used to screen the protein 105 database of each species with hmmsearch in HMMER 3.0 ( $E$-value $<0.01)$. Based on BLASTP 106 and hmmsearch analyses, the candidate thiolase genes were identified and subsequently checked 107 by conserved domain search (CD-Search) in NCBI and hmmscan against Pfam database ( $E$-value $108<1 \mathrm{e}-5)$. The candidate sequences that have Thiolase_N and/or Thiolase_C domains were 109 recognized as thiolases. Those identified thiolases were used as new queries to perform BLASTP 110 search against the protein database of each species until no more novel loci can be found. All the 111 validated thiolase genes were used for further analysis.

112 Phylogenetic analysis 
113 The protein sequences of thiolases from 20 insects, $H$. sapiens and $M$. tuberculosis were aligned 114 using MUSCLE (Edgar 2004). Positions that had a high percentage of gaps (>70\%) were trimmed. 115 The handled alignment of protein sequences was used for checking the most suitable model of 116 evolution by ProtTest 3.2 (Darriba et al. 2011). Maximum-likelihood (ML) trees were 117 reconstructed using RAxML version 8.2.12 (Stamatakis 2014) with the most suitable model 118 (PROTGAMMAVTF) and 500 bootstrap replicates. FigTree v1.4.3

119 (http://tree.bio.ed.ac.uk/software/figtree/) was used for plotting the final phylogenetic tree. The 120 clustering and classification of the thiolase sequences in the ML tree were done using known 121 functional properties of H. sapiens and M. tuberculosis (Anbazhagan et al. 2014).

122 Chromosome distribution, gene structure, and syntenic analysis

123 To localize the thiolase genes on chromosomes, B. mori, H. melpomene, D. melanogaster, T. 124 castaneum, and $A$. mellifera were selected because their genome sequences have been assembled 125 into chromosomes. Based on the GFF (General Feature Format) file of each species, every thiolase 126 gene was mapped to the corresponding chromosomes. Using protein sequences of the thiolases, 127 the precise exon/intron structures were generated through BLAT search against the genome 128 sequences with Scipio server (https:/www.webscipio.org/). The synteny events between two 129 species were detected by Multiple Collinearity Scan toolkit (MCScanX) with the default 130 parameters (Wang et al. 2012). The syntenic map of B. mori and H. melpomene was constructed 131 with family_circle_plotter.java in MCScanX software.

\section{Molecular modeling of protein structure}

133 The three-dimensional (3D) structure prediction of insect thiolases was conducted using the 134 homology modeling method. Structures of T1-, T2-, CT-, AB-, TFE-, and SCP2-thiolases were 135 predicted on-line at the SWISS-MODEL Interactive Workspace (Arnold et al. 2006). The known 136 protein that has the highest sequence similarity to the thiolase to be analyzed is used for homology 137 modeling. The predicted models of monomer and multimer were visualized in Swiss-PdbViewer 138 4.1.0 (Guex \& Peitsch 1997). To understand the 3D structural similarities among the insect 139 thiolases, all the other structures were compared with BmorT2 using magic fit algorithm in Swiss140 PdbViewer, respectively. The root-mean-square distance (RMSD) values were calculated to 141 express the structural similarity. The lower value of RMSD means higher similarity between two 142 structures (Carugo \& Pongor 2001).

143 Reverse transcription-polymerase chain reaction (RT-PCR) 
144 The various tissues on day 3 of fifth-instar larvae were dissected in the silkworm. The sex 145 pheromone glands (PGs) from 5 individuals were used as one sample at each developmental stage. 146 All the samples were preserved in RNAlater (Ambion, 98 Austin, USA) and stored at $-80{ }^{\circ} \mathrm{C}$ for 147 RNA isolation. Total RNA was extracted using Trizol reagent (Invitrogen, USA). The first strand 148 of cDNA was synthesized by M-MLV reverse transcriptase following the manufacturer's 149 instructions (Promega, USA). RT-PCR primers were listed in Table S2. The silkworm RpL3 gene 150 was used as an internal control for relative quantitative analysis of RT-PCR. PCRs were performed 151 with the following cycling parameters: $95^{\circ} \mathrm{C}$ for 3 minutes (min), followed by 25 cycles of 30 152 seconds (s) at $95^{\circ} \mathrm{C}, 30 \mathrm{~s}$ annealing (temperatures listed in Table S2) and $30 \mathrm{~s}$ extension $\left(72{ }^{\circ} \mathrm{C}\right)$, 153 and a final extension at $72{ }^{\circ} \mathrm{C}$ for $10 \mathrm{~min}$. The amplification products were monitored on $1.5 \%$ 154 agarose gels.

155 Results

\section{Genome-wide identification and phylogeny of insect thiolase proteins}

157 To identify thiolases in insects, human and M. tuberculosis thiolase protein sequences were used 158 as queries to perform homologous searches in whole genomes. In total, 137 thiolase genes were 159 identified in 20 insects from 7 orders, and the gene numbers of the species were ranged from 4 to 16015 (Table 1; Table S1). The thiolase protein sequences were used to reconstruct the maximum161 likelihood phylogenetic tree (Fig. 1). Based on the nomenclature rules in humans and $M$. 162 tuberculosis (Anbazhagan et al. 2014), each thiolase was named in insects. It was indicated that 163 insect thiolases were grouped 7 classes, namely CT, T1, T2, TFE, SCP2 (type-1), TFEL (type-2), 164 and AB (Table 1; Fig. 1). Relatively, the classification of insect thiolases was more similar to that 165 of the human than M. tuberculosis. Unlike humans, out of 20 insect species, only P. xuthus has 166 gene members in AB class. Interestingly, 5 out of 6 Lepidopteran insects have no CT-thiolase. Furthermore, TFEL (type-2) class was only detected in C. lectularius (Hemipter) and bacterium M. tuberculosis.

To understand the evolutionary mode, gene gain and loss of thiolases were analyzed. It was indicated that most of the gain and loss events were occurred in a certain species (Fig. 2). 171 Especially, P. xylostella (Lepidoptera), A. pisum (Hemipter), and P. xuthus (Lepidoptera) showed more duplications after or during the formation of the species, resulting in a total number of 15 , 173 12, and 10 genes, respectively. Except for the gene duplication of a single species lineage, the 174 common ancestor of D. alloeum, A. mellifera and B. impatiens showed 2 duplications (Fig. 2). 
175 This phenomenon was also noted in the clade of Hemipteran H. halys, C. lectularius, and A. pisum.

176 For those recent duplication genes, they were often phylogenetically closely related to its ancestral 177 genes (Fig. 1). Conversely, A. mellifera (Hymenoptera), H. halys (Hemipter), and Z. nevadensis

178 (Isoptera) presented 3, 3, and 2 gene losses during speciation, resulting in fewer genes in these 179 species. Generally, gene gain and loss rates are important for understanding the role of natural 180 selection and adaptation in shaping gene family sizes. For the species with more gene expansion, 181 whether these duplicated genes play roles in adapting to special habitats deserves further study.

\section{Gene structures of insect thiolase genes}

183 A comparative analysis of exon-intron structures was conducted for the 137 insect thiolase genes 184 (Fig. 3A and 3B; Fig. S2A). The insect thiolase genes have a different number of introns ranging 185 from 0 to 22 . It was indicated that only 12 genes have no intron, and 17 genes have only one intron, 186 accounting for $21.17 \%$ in total. The intronless genes were distributed in T2 (5), SCP2 (type-1) (2), 187 CT (2), AB (2), and TFEL (1). Previous studies revealed that introns can delay regulatory response 188 and are selected against in genes whose transcripts need to be adjusted quickly to meet 189 environmental challenges (Jeffares, Penkett \& Bahler 2008). The intronless thiolase genes and the genes contained fewer introns might play important roles in survival for environmental changes. In addition, the intron number and exon/intron structures of thiolase genes are very different, even the orthologous genes of different species in the same class have a large differentiation. It was suggested that the differentiation of the intron number may result in the diversification of thiolase gene structures in insects.

\section{Chromosome distribution and gene synteny}

196 In order to explore the chromosomal distribution of thiolase genes, five representative species were analyzed. It was indicated that most of the thiolase genes were randomly distributed on different chromosomes (Fig. S3A-E), for example, 6 thiolase genes were scattered on 4 chromosomes in $D$. melanogaster (Fig. S3A), which is similar to thiolase genes in the human (Anbazhagan et al. 2014). However, different members of a certain class are often tandem distribution, such as DmelSCP2 (type-1)-1 and DmelSCP2 (type-1)-2 in D. melanogaster (Fig. S3A) and BmorT1-1, BmorT1-2, and BmorT1-3 in B. mori (Fig. S3C). Meanwhile, we also detected the distribution of the 10 T1thiolase genes in P. xylostella and 7 CT-thiolase genes in A. pisum, which were distributed on several small unassembled scaffolds. Whether they are distributed in tandem, we still need to wait 
205 for the scaffold sequences to be integrated into the corresponding chromosome in future. In 206 general, tandem duplication might be the main mechanism for enlarging thiolase family in insects. 207 The syntenic relationships of thiolase genes were investigated among B. mori, H. melpomene, 208 D. melanogaster, T. castaneum, and A. mellifera because their genome sequences have been 209 assembled into chromosome levels. The results indicated that only four genes exhibited the 210 syntenic relationships between B. mori and H. melpomene, that is, BmorT2 and HmelT2, BmorTFE 211 and HmelTFE (Fig. S3F). Interestingly, except for the tandemly duplicated genes, amount of 212 thiolase genes often present orthologous relationships among insect, human, and M. tuberculosis 213 (Fig. 1). It was suggested that thiolase family is an ancient gene family. Even in insects, the age of 214 thiolase gene differentiation is relatively long. Thus, the discovery of fewer syntenic genes implies 215 that thiolases might mainly locate in some non-conserved genomic blocks (The Heliconius 216 Genome Consortium 2012).

\section{Subcellular localization of thiolase proteins}

218 Subcellular localization refers to the specific location of a certain protein or the expression product 219 of a certain gene in the cell. Protein subcellular localization is closely related to protein functions 220 (Pereto, Lopez-Garcia \& Moreira 2005; Wang et al. 2014). Only when the protein is positioned 221 correctly can it perform normal biological functions. In this study, subcellular localization of all 222 the 137 insect thiolase proteins was predicted by PSORT II server 223 (https://www.genscript.com/tools/psort), which were cytosolic, mitochondrial, or peroxisomal 224 enzymes (Fig. 3C; Fig. S2B). Generally, most of the TFE- and T2-thiolase proteins were located 225 in the mitochondrion, T1- and CT-thiolases were cytosolic, and SCP2-thiolases were peroxisomal 226 proteins. Previous studies suggested that the mitochondrial and peroxisomal thiolase proteins were 227 mainly involved in the fatty acid $\beta$-oxidation pathway (Pereto, Lopez-Garcia \& Moreira 2005), 228 and cytosolic localization was related to the biosynthesis of acetoacetyl-CoA (Kursula et al. 2005). 229 However, in a certain class of thiolase, there are always a few exceptions to the cellular location 230 in some species (Fig. 3C; Fig. S2B), which suggested that its function might have diverged during 231 evolution.

\section{Conserved domain characteristics and catalytic residues}

233 To identify the potential domains of insect thiolase proteins (Table S1), it was performed hmmscan 234 analysis in Pfam database. The results indicated that all the thiolases contained Thiolase_N and 235 Thiolase_C domains (Fig. 4A). In addition to the thiolase domains, SCP2-thioloase (type-1) has a 
236 typical sterol carrier protein 2 (SCP2) domain at C terminal. Unexpectedly, some of the members in 237 TFE, CT, and T2 classes contained a ketoacyl-synt (beta-ketoacyl synthase) domain within 238 Thiolase_N (Table S1). We carefully checked the alignments of hmmscan search. It was found 239 that the $E$ value was around the threshold 1e-5, and only about 50 amino acids can be aligned, 240 which are much shorter than 250 amino acids of the ketoacyl-synt domain (Pfam ID, PF00109). 241 Thus, thiolases may not contain the real ketoacyl-synt domain, and just show certain similarities 242 with it (Huang et al. 1998). Therefore, based on the domain characteristics, all the insect thiolase 243 encoding genes were classified as 2 groups (Fig. 4A).

244 The conserved sequence blocks of the 20 insects, humans, and M. tuberculosis were analyzed 245 (Fig. S4; Fig. 4B). The CxS-motif is the most important sequence fingerprint in the N-terminal 246 domain, which provides the nucleophilic cysteine (Zeng \& Li 2004; Mazet et al. 2011). Except for 247 some incomplete sequences, almost all the thiolases contained the cysteine residue (Fig. S4; Fig. 248 4B). The histidine of the GHP-motif contributes to the oxyanion hole of the thioester oxygen 249 (Merilainen et al. 2009). It was indicated that GHP-motif was highly conserved in insects, humans, 250 and M. tuberculosis (Fig. S4; Fig. 4B). The cysteine of CxGGGxG-motif provides the catalytic 251 residue of the active sites. Except for SCP2-thiolases, the catalytic cysteine was retained in almost 252 all the other thiolases (Fig. S4). In addition, the asparagine side chain of the NEAF-motif interacts 253 with important catalytic water (Mazet et al. 2011). However, NEAF-motif was replaced by HDCF254 motif in all of the SCP2-thiolases (Fig. S4; Fig. 4B). Based on the comparison of the sequence 255 fingerprints, it was indicated that the catalytic mechanisms of the insect thiolases might be similar 256 to that of thiolases from mammals and bacteria.

\section{Molecular modeling of insect thiolases}

258 In recent years, the crystal structures of some thiolases have been gradually resolved in bacteria, 259 fish, and mammals (Harijan et al. 2013; Kim et al. 2015; Xia et al. 2019). The high sequence 260 similarities ( $>60 \%$ ) may help to build more accurate 3D structures for the insect thiolases (Arnold 261 et al. 2006). Based on homology modeling using SWISS-MODEL Interactive Workspace, we 262 found that thiolase sequences within a class were very conserved among different organisms. For 263 instance, BmorTFE-thiolase and BmorSCP2-thiolase (type-1) shared 67.58\% and $61.63 \%$ identities with its corresponding modeling templates from human (PDB ID: 6dv2.1.A) and zebrafish (6hrv.2.A), respectively. In this study, the modeling structures of some representative 
267 detected with magic fit in Swiss-PdbViewer (Guex \& Peitsch 1997). The RMSD values were 268 ranged from $0.25 \AA$ to $0.91 \AA$ among BmorT2, BmorT1-1, DmelCT, and PxutAB-1, while 269 BmorTFE and BmorSCP2 (type-1) shared from $1.12 \AA$ to $2.01 \AA$ with the others (Table S3). It 270 was indicated that $\mathrm{T} 1, \mathrm{~T} 2, \mathrm{CT}$, and $\mathrm{AB}$ classes share more similar 3D structures than TFE-thiolase 271 and SCP2-thiolase (type-1) (Fig. 5A-F). This phenomenon is widespread in both humans and $M$. 272 tuberculosis (Harijan et al. 2013; Anbazhagan et al. 2014). For the quaternary structure, different 273 thiolases also have certain differences. For example, BmorT1-1 and BmorSCP2 (type-1) were 274 homo-tetramer and homo-dimer, respectively (Fig. 5G and 5F). The results of 3D structural 275 modeling showed that different classes of thiolase genes still present some extent divergence in 276 tertiary or quaternary structures.

277 SCP2-thiolase (type-1) was widely distributed in insects, mammals, and bacteria (Table 1). 278 One single structural gene referred to as the sterol carrier protein $\mathrm{x}(S C P x)$ gene encodes a full279 length protein comprised of 3-oxoacyl-CoA thiolase (known as SCP2-thiolase) and sterol carrier 280 protein 2 (Seedorf et al. 1994; Gallegos et al. 2001). The C-terminal SCP2-domain containing the 281 peroxisomal targeting signal is needed for the targeting of full-length SCPx into the peroxisomes.

282 The SCP2-thiolase and SCP2 protein are produced from SCPx via proteolytic cleavage by 283 peroxisomal proteases (Seedorf et al. 1994). Based on the homology modeling, the tertiary and 284 quaternary structures of mature SCP2-thiolase (type-1) protein were presented in Fig. 5F and 5H, 285 respectively. For insect SCP2-thiolases, the canonical CxGGGxG-motif is also absent, and the 286 NEAF-motif has been replaced by HDCF-motif (Fig. 4B). The previous studies indicated that HDCF-motif might provide the catalytic cysteine in bacteria, mammals, and fish (Harijan et al. 2013; Kiema et al. 2019). Based on the structural modeling, the cysteine of HDCF-motif is very close to the other two catalytic sites in protein spatial conformation (Fig. 5F). Therefore, the catalytic cysteine of the insect SCP2-thiolases might be not provided by CxGGGxG-motif but

\section{Expression profile and potential functional diversity}

293 To understand the potential functional diversity of the insect thiolases, the silkworm, B. mori, was 294 used as a model organism to perform expression profile analysis in the various tissues and sex pheromone glands (PGs) at different developmental stages. In the silkworm, genome-wide microarray with 22,987 oligonucleotides was designed and surveyed the gene expression profiles 
298 were found its corresponding probes (Fig. 6A). The microarray data indicated that BmorSCP2 299 (type-1), BmorT2, BmorTFE, and BmorT1-1 have expression signals at least one of the 9 tissues. 300 Relatively, BmorT2 and BmorT1-1 showed ubiquitous expressions. Meanwhile, the expression 301 profiles of the four genes were similar between females and males, respectively.

To validate the expression profiles of the silkworm thiolase genes, the mixed male and 303 female tissues were used to perform RT-PCR validation on day 3 of the fifth-instar larvae (Fig.

304

305

306

307

308

309

310

311

312

\section{3}

314

315

316

317

318

319

320

321

322

323

324

325

326

327

328 6B). In total, 5 out of the 6 thiolase genes presented expression evidence. Relatively, BmorT1-2 and BmorTFE showed predominant expressions in hemocyte and head, respectively (Fig. 6B), while BmorT1-1 was widely expressed in various tissues. In addition, sex pheromone glands of different developmental stages were used to detect the expressions of thiolase genes (Fig. 6C). Four thiolase genes presented expression signals in the silkworm PGs. Relatively, BmorT1-1 showed the highest expression on day 8 of pupae. BmorTFE, BmorT2, and BmorSCP2 (type-1) presented expressions at all the developmental stages. Interestingly, the expression levels of all three genes were declined in the mated female PGs (Fig. 6C). These expression analyses might help us understand the functional divergence of the thiolase genes in the silkworm.

\section{Discussion}

Thiolases are widely distributed in all organisms and are essential for a range of metabolic pathways. With the development of sequencing technology, it provides the possibility for us to identify and compare insect thiolase at the whole genome level. In this study, 137 thiolase genes were identified in the 20 representative species from 7 insect orders (Table 1; Table S1). The insect thiolases were mainly classified into five classes, including CT-thiolase, T1-thiolase, T2-thiolase, TFE-thiolase, and SCP2-thiolase. It was indicated that P. xylostella, A. pisum, and P. xuthus showed more duplications, resulting in a total number of 15,12 , and 10 genes, respectively. $Z$. nevadensis and $H$. melpomene have the least number of genes (Table 1). In addition to a certain differentiation in the number of genes, Thiolase_N or Thiolase_C domains of 9 thiolase genes were missing (Table S1). It is worth noting that the quality of the genome may have a certain impact on the number of genes and the integrity of gene structures. Whether the incomplete thiolase genes were pseudogenes or not (Table S1) needs further verification by the high-quality genome in the future.

Two groups of thiolases were identified in animals: 3-oxoacyl-CoA thiolase and acetoacetylCoA thiolase, which participates in different catabolic (fatty acid oxidation and bile acid 
329 formation) and anabolic (cholesterogenesis, ketone body synthesis, fatty acid elongation) 330 processes (Antonenkov, Van Veldhoven \& Mannaerts 1999). It is well known that cholesterol is a 331 precursor of molting hormone, 20-hydroxyecdysone (20E), and is a structural component of cell 332 membranes (Gilbert, Rybczynski \& Warren 2002). Due to the lack of squalene monooxygenase 333 and lanosterol synthase for the synthesis of cholesterol, insects can not autonomously synthesize 334 the 20E precursor (Guo et al. 2009). Alternatively, insects can obtain cholesterol or other sterols 335 from their diet to meet the needs of growth and development. In Spodoptera litura, one sterol 336 carrier protein $\mathrm{x}(S C P x)$ gene encoding a sterol carrier protein 2 and a 3-oxoacyl-CoA thiolase 337 known as SCP2-thiolase (type-1) showed predominant expression in the midgut, and its coding 338 SCP2 was involved in the absorption and transport of cholesterol (Guo et al. 2009). In the silkworm, SCPX gene has been cloned (Gong et al. 2006). It presented expressions in the midgut, fat body, and head on day 3 of the fifth-instar larvae in the silkworm (Fig. 6B), which suggested

341

342 343

344

345

346

347

348

349

350

351

352 353 354 355 356

357 that the SCP2 protein might have a similar function with that of S. litura (Guo et al. 2009). More important, the SCP2-thiolase (type-1) encoded by SCPx plays a crucial role in the oxidation of the branched side chain of cholesterol to form bile acids in vertebrates (Ferdinandusse et al. 2000), while the physiological role has not been characterized in insects. Fortunately, the expression of the SCP2-thiolase (type-1) has also been detected in the prothoracic glands of Spodoptera littoralis, which are the main tissue producing the insect molting hormone (Takeuchi et al. 2004).

Thus, whether SCP2-thiolase (type-1) of the silkworm and other insects play role in the oxidation of cholesterol and participates in ecdysone synthesis needs further study.

In insects, juvenile hormone $(\mathrm{JH})$ is an important regulator for growth and development (Kinjoh et al. 2007) and several thiolases have been cloned and suggested to be related to JH biosynthesis (Kinjoh et al. 2007; Zhu et al. 2016; Zhang et al. 2017). Acetoacetyl-CoA thiolase catalyzes two molecules of acetyl-CoA to form acetoacetyl-CoA, which is the first enzyme in JH biosynthesis (Kinjoh et al. 2007; Zhang et al. 2017). The candidate acetoacetyl-CoA thiolases related to JH biosynthesis were cloned in B. mori and Helicoverpa armigera (Kinjoh et al. 2007; Zhang et al. 2017). In this study, those two acetoacetyl-CoA thiolase genes were classified as T2thiolases (BmorT2 and HarmT2), and they shared high sequence identities with the other T2thiolases (Table S4). For example, BmorT2-thiolase shared $82.71 \%$ sequence identity with HarmT2. In $H$. armigera, temporal expressions of HarmT2-thiolase keep pace with JH fluctuations, and its expression can be inhibited by a juvenile hormone analog (Zhang et al. 2017). 
360 The expression of BmorT2-thiolase was relatively abundant in the head where the JH synthetic 361 gland, corpora allata (CA), is located (Fig. 6A and 6B). Interestingly, we found BmorTFE- and 362 BmorT1-1-thiolase also showed high expressions in the larval head (Fig. 6A). In humans, TFE363 and T1-thiolases catalyze thiolytic cleavage of 3-ketoacyl-CoA into acetyl-CoA and acyl-CoA 364 (Anbazhagan et al. 2014; Xia et al. 2019). However, T1-thiolase has been found synthetic and 365 degradative activities in Ostrinia scapulalis (Lepidoptera: Crambidae). Therefore, whether T2-, 366 TFE- and T1-thiolases were involved in JH biosynthesis is still worthy of experimental validation. 367 Acetyl-CoA is often used as the initial precursor for sex pheromone biosynthesis in insects 368 (Matsumoto 2010). Degradative thiolases may supplement with sufficient acetyl-CoA for sex 369 pheromone synthesis (Brabcova et al. 2015). In this study, expression profiles of the thiolase genes 370 were detected in the sex pheromone glands at different developmental stages in the silkworm (Fig. 371 6C). Relatively, BmorSCP2 (type-1) maintains a high level of expression in the PGs on day 4 of 372 pupae to 24-h-old virgin female moth. However, its expression level was sharply declined in the 373 mated female PGs (Fig. 6C). The previous study suggested that an over 6-h mating duration can 374 terminate the sex pheromone production in the silkworm (Ando et al. 1996). The expression pattern 375 of BmorSCP2 (type-1) was consistent with sex pheromone production (Matsumoto 2010), which 376 suggested that it might be involved in sex pheromone biosynthesis. Generally, it is tempting to 377 assume that a thiolase expressed in a specific tissue might obtain a specific role. Thus, the 378 functional diversification and physiological roles of insect thiolases need yet further experimental 379 validation.

\section{Conclusions}

381 In the present study, genome-wide identification of the thiolase gene family was conducted for the 382 first time in multiple insect genomes. A total of 137 thiolase genes were identified in 20 insects 383 from 7 orders. About $80 \%$ of the thiolase genes have 2 or more introns, and its exon/intron 384 structures reserve diversification. Based on the prediction, all the thiolase proteins are located in 385 the mitochondria, cytosol, or peroxisome, and thiolases of the same class often have similar 386 cellular localization. Four highly conserved sequence fingerprints were found in the insect thiolase 387 proteins, including CxS-, NEAF-, GHP-, and CxGGGxG-motifs. Homology modeling analysis 388 indicated that 3D structures of the insect thiolases share similar to mammals, fishes, and microorganisms. Expression pattern analysis suggested some thiolase genes may be involved in 
390

391

392

393

394

395

396

397

398

399

400

401

402

403

404

405

406

407

408

409

410

411

412

413

414

415

416

417

418

419

420

421

422

423

steroid metabolism, $\mathrm{JH}$, and sex pheromone biosynthesis pathways in B. mori. These results might provide valuable information for the functional exploration of thiolase proteins in insects.

\section{Acknowledgements}

This study was supported by the Initiation Fund (No. 15E022) and Teaching Reform Research Project (No. Jgxmyb18151) of China West Normal University. The author sincerely thanks the anonymous reviewers for their affirmation and constructive comments on the manuscript.

\section{References}

Anbazhagan P, Harijan RK, Kiema TR, Janardan N, Murthy MR, Michels PA, Juffer AH, Wierenga RK. 2014. Phylogenetic relationships and classification of thiolases and thiolase-like proteins of Mycobacterium tuberculosis and Mycobacterium smegmatis. Tuberculosis (Edinb) 94:405-412.

Ando T, Kasuga K, Yajima Y, Kataoka H, Suzuki A. 1996. Termination of sex pheromone production in mated females of the silkworm moth. Archives of Insect Biochemistry and Physiology 31:207-218.

Antonenkov VD, Van Veldhoven PP, Mannaerts GP. 1999. The growing family of peroxisomal thiolases. Lipids 34 Suppl:S157.

Antonenkov VD, Van Veldhoven PP, Waelkens E, Mannaerts GP. 1999. Comparison of the stability and substrate specificity of purified peroxisomal 3-oxoacyl-CoA thiolases A and B from rat liver. Biochimica Et Biophysica Acta-Molecular and Cell Biology of Lipids 1437:136-141.

Antonenkov VD, VanVeldhoven PP, Waelkens E, Mannaerts GP. 1997. Substrate specificities of 3-oxoacylCoA thiolase A and sterol carrier protein 2/3-oxoacyl-CoA thiolase purified from normal rat liver peroxisomes - Sterol carrier protein 2/3-oxoacyl-CoA thiolase is involved in the metabolism of 2methyl-branched fatty acids and bile acid intermediates. Journal of Biological Chemistry 272:2602326031 .

Arnold K, Bordoli L, Kopp J, Schwede T. 2006. The SWISS-MODEL workspace: a web-based environment for protein structure homology modelling. Bioinformatics 22:195-201.

Brabcova J, Demianova Z, Kindl J, Pichova I, Valterova I, Zarevucka M. 2015. Characterisation of acetylCoA thiolase: the first enzyme in the biosynthesis of terpenic sex pheromone components in the labial gland of Bombus terrestris. Chembiochem 16:1047-1051.

Clinkenbeard KD, Sugiyama T, Moss J, Reed WD, Lane MD. 1973. Molecular and catalytic properties of cytosolic acetoacetyl coenzyme A thiolase from avian liver. Journal of Biological Chemistry 248:22752284.

Darriba D, Taboada GL, Doallo R, Posada D. 2011. ProtTest 3: fast selection of best-fit models of protein evolution. Bioinformatics 27:1164-1165.

Edgar RC. 2004. MUSCLE: multiple sequence alignment with high accuracy and high throughput. Nucleic Acids Research 32:1792-1797. 
424

425

426

427

428

429

430

431

432

433

434

435

436

437

438

439

440

441

442

443

444

445

446

447

448

449

450

451

452

453

454

455

456

457

458

Ferdinandusse S, Denis S, van Berkel E, Dacremont G, Wanders RJ. 2000. Peroxisomal fatty acid oxidation disorders and $58 \mathrm{kDa}$ sterol carrier protein X (SCPx). Activity measurements in liver and fibroblasts using a newly developed method. Journal of Lipid Research 41:336-342.

Fujii T, Ito K, Katsuma S, Nakano R, Shimada T, Ishikawa Y. 2010. Molecular and functional characterization of an acetyl-CoA acetyltransferase from the adzuki bean borer moth Ostrinia scapulalis (Lepidoptera: Crambidae). Insect Biochemistry and Molecular Biology 40:74-78.

Fukao T. 2002. Acetoacetyl-CoA thiolase (mitochondrial). Wiley encyclopedia of molecular medicine, Vol. 5. John Wiley \& Sons, Inc., New York, pp. 6-9.

Fukao T, Song XQ, Mitchell GA, Yamaguchi S, Sukegawa K, Orii T, Kondo N. 1997. Enzymes of ketone body utilization in human tissues: Protein and messenger RNA levels of succinyl-coenzyme A (CoA):3ketoacid CoA transferase and mitochondrial and cytosolic acetoacetyl-CoA thiolases. Pediatric Research 42:498-502.

Gallegos AM, Atshaves BP, Storey SM, Starodub O, Petrescu AD, Huang H, McIntosh AL, Martin GG, Chao H, Kier AB, Schroeder F. 2001. Gene structure, intracellular localization, and functional roles of sterol carrier protein-2. Progress in Lipid Research 40:498-563.

Gilbert LI, Rybczynski R, Warren JT. 2002. Control and biochemical nature of the ecdysteroidogenic pathway. Annual Review of Entomology 47:883-916.

Gong J, Hou Y, Zha XF, Lu C, Zhu Y, Xia QY. 2006. Molecular cloning and characterization of Bombyx mori sterol carrier protein $\mathrm{x} /$ sterol carrier protein 2 (SCPx/SCP2) gene. DNA Sequence 17:326-333.

Guex N, Peitsch MC. 1997. SWISS-MODEL and the Swiss-PdbViewer: an environment for comparative protein modeling. Electrophoresis 18:2714-2723.

Guo XR, Zheng SC, Liu L, Feng QL. 2009. The sterol carrier protein 2/3-oxoacyl-CoA thiolase (SCPx) is involved in cholesterol uptake in the midgut of Spodoptera litura: gene cloning, expression, localization and functional analyses. BMC Molecular Biology 10:102.

Harijan RK, Kiema TR, Karjalainen MP, Janardan N, Murthy MR, Weiss MS, Michels PA, Wierenga RK. 2013. Crystal structures of SCP2-thiolases of Trypanosomatidae, human pathogens causing widespread tropical diseases: the importance for catalysis of the cysteine of the unique HDCF loop. Biochemical Journal 455:119-130.

Houten SM, Wanders RJA. 2010. A general introduction to the biochemistry of mitochondrial fatty acid betaoxidation. Journal of Inherited Metabolic Disease 33:469-477.

Huang W, Jia J, Edwards P, Dehesh K, Schneider G, Lindqvist Y. 1998. Crystal structure of beta-ketoacylacyl carrier protein synthase II from E.coli reveals the molecular architecture of condensing enzymes. EMBO Journal 17:1183-1191.

Igual JC, Gonzalez-Bosch C, Dopazo J, Perez-Ortin JE. 1992. Phylogenetic analysis of the thiolase family. Implications for the evolutionary origin of peroxisomes. Journal of Molecular Evolution 35:147-155.

Peer] reviewing PDF | (2020:08:52282:3:0:NEW 23 Oct 2020) 
459

460

461

462

463

464

465

466

467

468

469

470

471

472

473

474

475

476

477

478

479

480

481

482

483

484

485

486

487

488

489

490

491

492

Jeffares DC, Penkett CJ, Bahler J. 2008. Rapidly regulated genes are intron poor. Trends in Genetics 24:375378.

Kiema TR, Thapa CJ, Laitaoja M, Schmitz W, Maksimainen MM, Fukao T, Rouvinen J, Janis J, Wierenga RK. 2019. The peroxisomal zebrafish SCP2-thiolase (type-1) is a weak transient dimer as revealed by crystal structures and native mass spectrometry. Biochemical Journal 476:307-332.

Kim S, Jang YS, Ha SC, Ahn JW, Kim EJ, Lim JH, Cho C, Ryu YS, Lee SK, Lee SY, Kim KJ. 2015. Redox-switch regulatory mechanism of thiolase from Clostridium acetobutylicum. Nature Communications 6:8410.

Kinjoh T, Kaneko Y, Itoyama K, Mita K, Hiruma K, Shinoda T. 2007. Control of juvenile hormone biosynthesis in Bombyx mori: cloning of the enzymes in the mevalonate pathway and assessment of their developmental expression in the corpora allata. Insect Biochemistry and Molecular Biology 37:808-818.

Kursula P, Sikkila H, Fukao T, Kondo N, Wierenga RK. 2005. High resolution crystal structures of human cytosolic thiolase (CT): A comparison of the active sites of human CT, bacterial thiolase, and bacterial KAS I. Journal of Molecular Biology 347:189-201.

Masamune S, Palmer MAJ, Gamboni R, Thompson S, Davis JT, Williams SF, Peoples OP, Sinskey AJ, Walsh CT. 1989. Bio-Claisen condensation catalyzed by thiolase from Zoogloea ramigera. active-site cysteine residues. Journal of the American Chemical Society 111:1879-1881.

Matsumoto S. 2010. Molecular mechanisms underlying sex pheromone production in moths. Bioscience, Biotechnology, and Biochemistry 74:223-231.

Mazet M, Harijan RK, Kiema TR, Haapalainen AM, Morand P, Morales J, Bringaud F, Wierenga RK, Michels PA. 2011. The characterization and evolutionary relationships of a trypanosomal thiolase. International Journal for Parasitology 41:1273-1283.

Merilainen G, Poikela V, Kursula P, Wierenga RK. 2009. The thiolase reaction mechanism: the importance of Asn 316 and His 348 for stabilizing the enolate intermediate of the Claisen condensation. Biochemistry 48:11011-11025.

Modis Y, Wierenga RK. 2000. Crystallographic analysis of the reaction pathway of Zoogloea ramigera biosynthetic thiolase. Journal of Molecular Biology 297:1171-1182.

Carugo O, Pongor S. 2001. A normalized root-mean-square distance for comparing protein three-dimensional structures. Protein Science 10:1470-1473.

Pereto J, Lopez-Garcia P, Moreira D. 2005. Phylogenetic analysis of eukaryotic thiolases suggests multiple proteobacterial origins. Journal of Molecular Evolution 61:65-74.

Schiedl AC, Oeljeklaus S, Minihan P, Dyer JH. 2004. Cloning, expression, and purification of glyoxysomal 3-oxoacyl-CoA thiolase from sunflower cotyledons. Protein Expression and Purification 33:25-33. 
493

494

495

496

497

498

499

500

501

502

503

504

505

506

507

508

509

510

511

512

513

514

515

516

517

518

519

520

521

522

523

524

525

526

527

Seedorf U, Brysch P, Engel T, Schrage K, Assmann G. 1994. Sterol carrier protein X is peroxisomal 3-oxoacyl coenzyme A thiolase with intrinsic sterol carrier and lipid transfer activity. Journal of Biological Chemistry 269:21277-21283.

Stamatakis A. 2014. RAxML version 8: a tool for phylogenetic analysis and post-analysis of large phylogenies. Bioinformatics 30:1312-1313.

Takeuchi H, Chen JH, Jenkins JR, Bun-Ya M, Turner PC, Rees HH. 2004. Characterization of a sterol carrier protein 2/3-oxoacyl-CoA thiolase from the cotton leafworm (Spodoptera littoralis): a lepidopteran mechanism closer to that in mammals than that in dipterans. Biochemical Journal 382:93100.

The Heliconius Genome Consortium 2012. Butterfly genome reveals promiscuous exchange of mimicry adaptations among species. Nature 487:94-98.

Thompson S, Mayerl F, Peoples OP, Masamune S, Sinskey AJ, Walsh CT. 1989. Mechanistic studies on beta-ketoacyl thiolase from Zoogloea ramigera: identification of the active-site nucleophile as Cys89, its mutation to Ser89, and kinetic and thermodynamic characterization of wild-type and mutant enzymes. Biochemistry 28:5735-5742.

Vogel H, Heidel AJ, Heckel DG, Groot AT. 2010. Transcriptome analysis of the sex pheromone gland of the noctuid moth Heliothis virescens. BMC Genomics 11:29.

Wang YP, Tang HB, DeBarry JD, Tan X, Li JP, Wang XY, Lee TH, Jin HZ, Marler B, Guo H, Kissinger JC, Paterson AH. 2012. MCScanX: a toolkit for detection and evolutionary analysis of gene synteny and collinearity. Nucleic Acids Research 40:e49.

Wang Z, Zou Q, Jiang Y, Ju Y, Zeng XX. 2014. Review of protein subcellular localization prediction. Current Bioinformatics 9:331-342.

Xia C, Fu Z, Battaile KP, Kim JP. 2019. Crystal structure of human mitochondrial trifunctional protein, a fatty acid beta-oxidation metabolon. Proceedings of the National Academy of Sciences of the United States of America 116:6069-6074.

Xia Q, Cheng D, Duan J, Wang G, Cheng T, Zha X, Liu C, Zhao P, Dai F, Zhang Z, He N, Zhang L, Xiang Z. 2007. Microarray-based gene expression profiles in multiple tissues of the domesticated silkworm, Bombyx mori. Genome Biology 8:R162.

Xia Q, Li S, Feng Q. 2014. Advances in silkworm studies accelerated by the genome sequencing of Bombyx mori. Annual Review of Entomology 59:513-536.

Zeng J, Li D. 2004. Expression and purification of His-tagged rat mitochondrial medium-chain acyl-CoA dehydrogenase wild-type and Arg256 mutant proteins. Protein Expression and Purification 37:472-478.

Zhang W, Ma L, Xiao H, Liu C, Chen L, Wu S, Liang G. 2017. Identification and characterization of genes involving the early step of Juvenile Hormone pathway in Helicoverpa armigera. Scientific Repports 7:16542.

PeerJ reviewing PDF | (2020:08:52282:3:0:NEW 23 Oct 2020) 
528 Zhu J, Khalil SM, Mitchell RD, Bissinger BW, Egekwu N, Sonenshine DE, Roe RM. 2016. Mevalonate529 farnesal biosynthesis in ticks: comparative synganglion transcriptomics and a new perspective. PLoS One 11:e0141084. 


\section{Figure 1}

Fig. 1. Phylogenetic tree of insect thiolases using the maximum-likelihood (ML) method.

The thiolases of human and $M$. tuberculosis were shown by stars and dots, respectively. The bootstrap values higher than $50 \%$ were dotted on the nodes. Lep: Lepidoptera; Dip: Diptera; Col: Coleoptera; Hem: Hemipter; Hym: Hymenoptera; Pht: Phthiraptera; Iso: Isoptera. The accession numbers following each gene name were presented. The information ENSANGP within accession numbers of $A$. gambiae was omitted. 


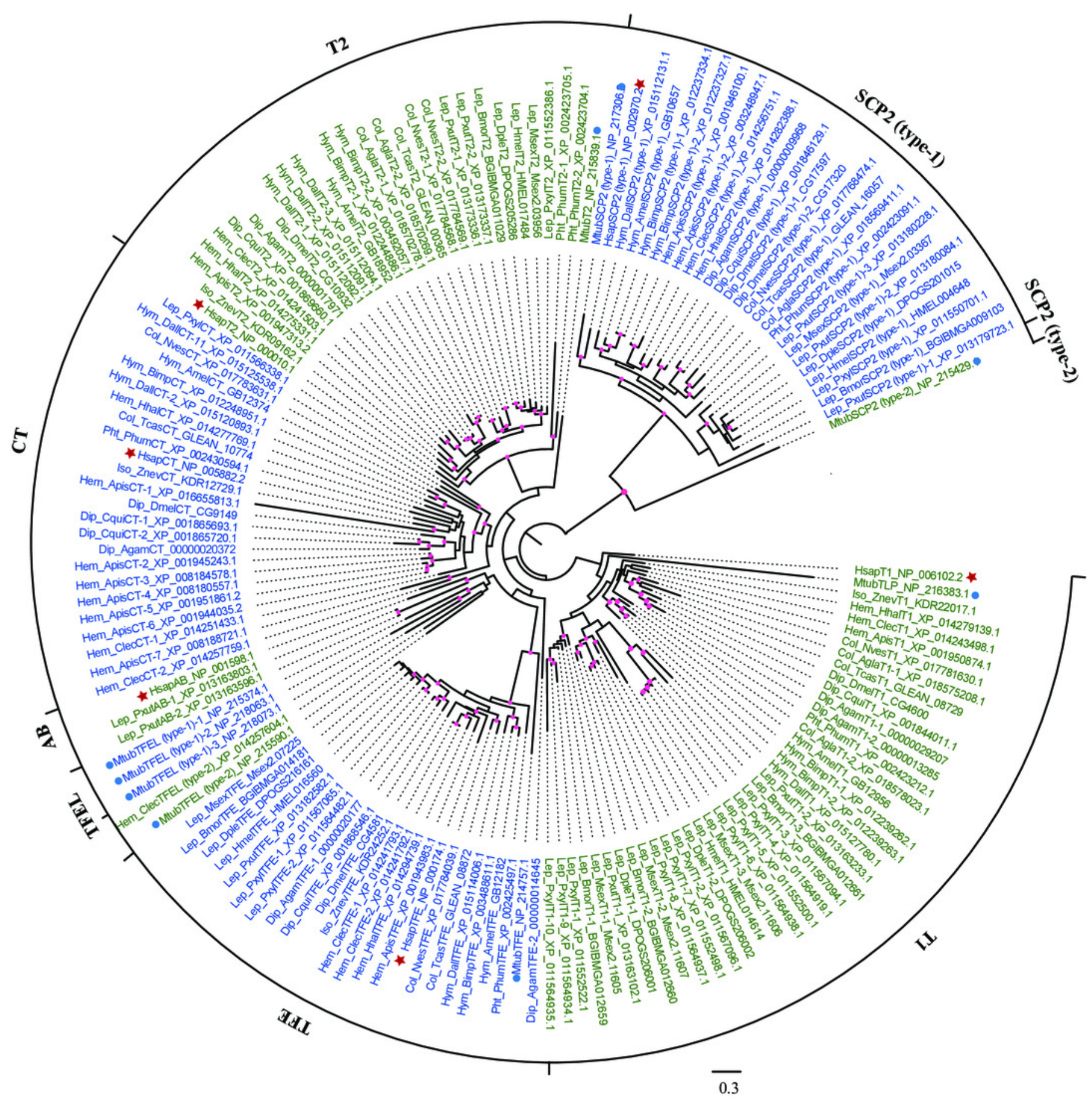




\section{Figure 2}

Fig. 2. Gene gain and loss analysis of the thiolase gene family in insect genomes.

The species tree was obtained from timetree database ( $\mathrm{http}: / / w w w . t i m e t r e e . o r g /$ ). Gain and loss analysis was conducted by Notung-2.9 software with default parameters. The orange and green vertical bars on branch presented gene gain and loss, respectively. The number in each node is gene count. Gene number of each species was presented in brackets.

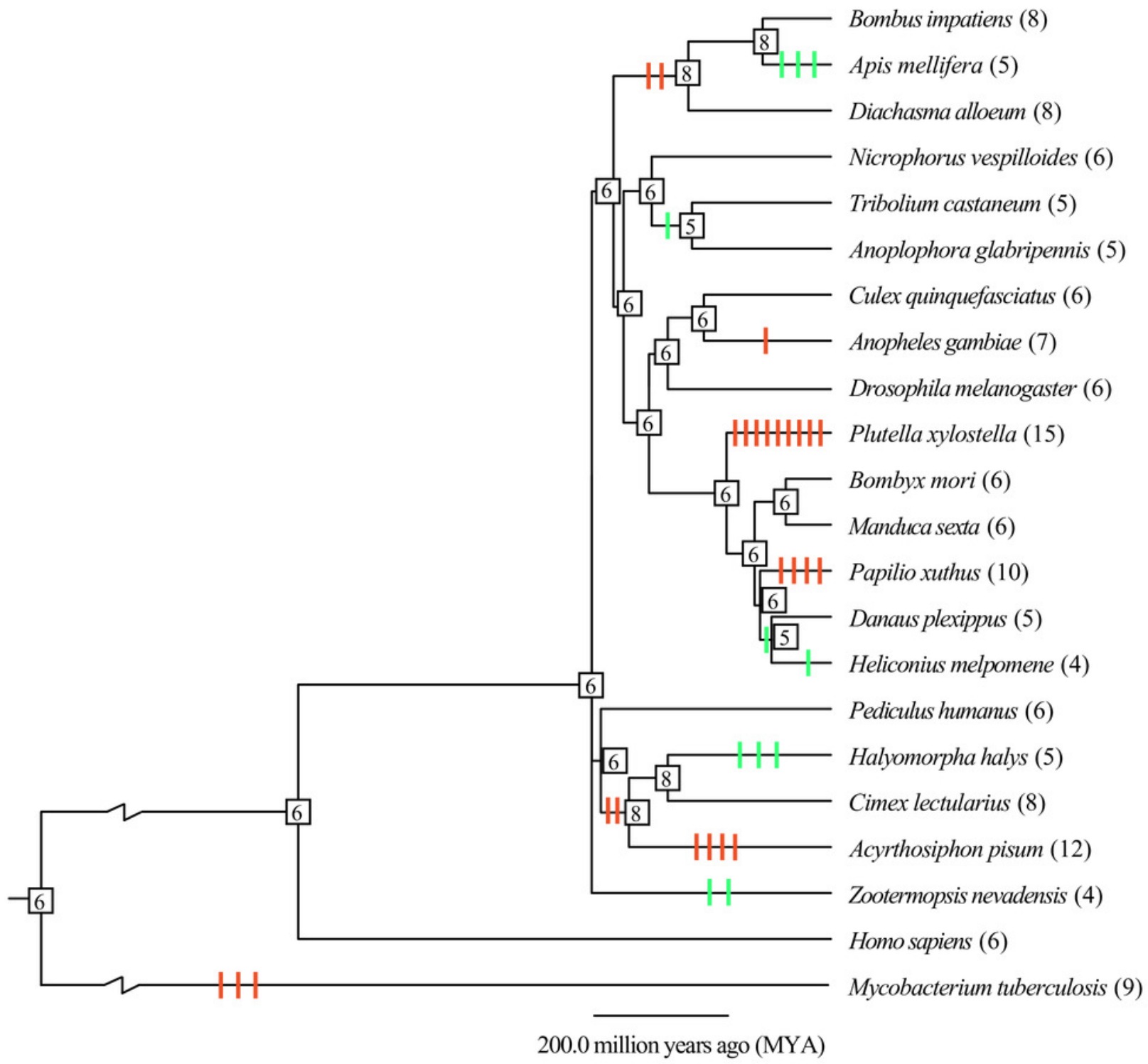




\section{Figure 3}

Fig. 3. Exon/intron structure and subcelluar localization analyses.

(A) The maximum-likelihood phylogenetic tree of thiolase proteins from 7 representative insects. The bootstrap values higher than 50\% were dotted on the nodes. (B) Exon/intron structure analysis of the thiolase genes. (C) Subcelluar localization analysis of thiolase proteins. It was predicted by PSORT II server (https://www.genscript.com/tools/psort). Mit: mitochondrion; Cyt: cytosol; Pox: peroxisome. 
(A) Phylogenetic tree

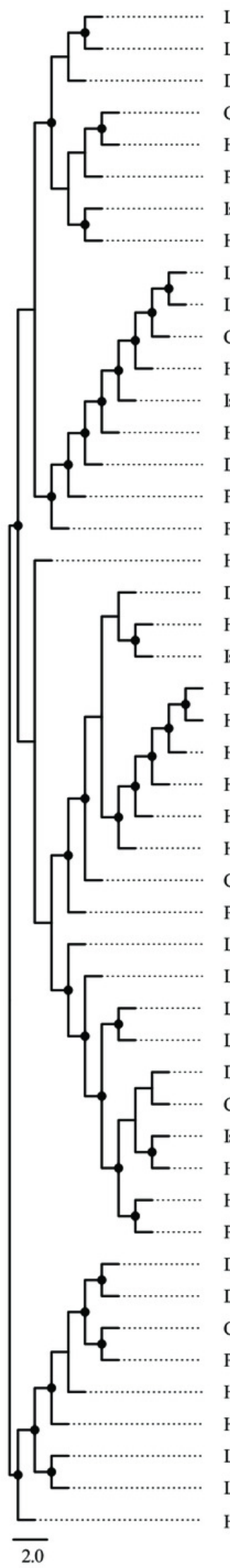

Lep_BmorTFE

Lep_HmelTFE

Dip_DmelTFE

Col_TcasTFE

Hym_AmelTFE

Pht PhumTFE

Iso_ZnevTFE

Hem_ApisTFE

Lep_BmorT2

Lep_HmelT2

Col_TcasT2

Hym_AmelT2

Iso_ZnevT2

Hem_ApisT2

Dip_DmelT2

Pht PhumT2-2

Pht PhumT2-1

Hem_ApisCT-7

Dip_DmelCT

Hym_AmelCT

Iso_ZnevCT

Hem_ApisCT-6

Hem_ApisCT-5

Hem_ApisCT-4

Hem_ApisCT-2

Hem_ApisCT-3

Hem_ApisCT-1

Col_TcasCT

Pht PhumCT

Lep_BmorT1-3

Lep_BmorT1-2

Lep_BmorT1-1

Lep_HmelT1

Dip_DmelT1

Col_TcasT1

Iso_ZnevT1

Hem_ApisT1

Hym_AmelT1

Pht PhumT1

Dip_DmelSCP2 (type-1)-1 1000bos

Dip_DmelSCP2 (type-1)-2

Col_TcasSCP2 (type-1)

Pht_PhumSCP2 (type-1)

Hem_ApisSCP2 (type-1)-1

Hym_AmelSCP2 (type-1)

Lep_BmorSCP2 (type-1)

Lep_HmelSCP2 (type-1)

Hem_ApisSCP2 (type-1)-2

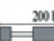

$100 \mathrm{pss}$

100 tops. robops

$2000 \mathrm{ks}$
(B) Gene structure analysis

$\square$ Exon (ex.) $=$ Intron (in.)
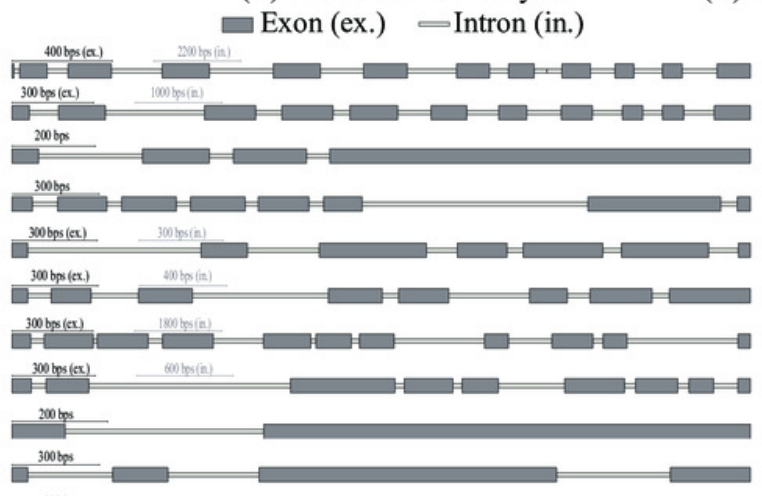

300bs(x)

${ }^{30 \text { bog }(a)}$

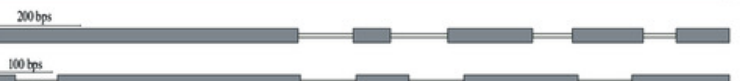

tototestex)

$200 \mathrm{bx}$

30 bos(ax)

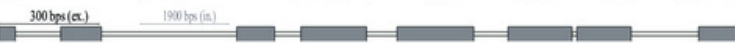

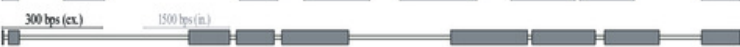

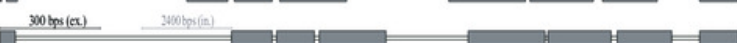

300ps(ser)

solos (ax)

xompops(ax)

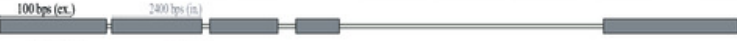

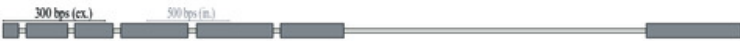

sobops

300bos(tex)

$\stackrel{\text { sobobsica) }}{\mathrm{r}} \longrightarrow$

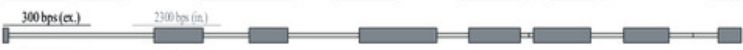

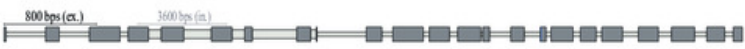

$\stackrel{300 \text { tops }(3 x)}{\square} \square \longrightarrow$

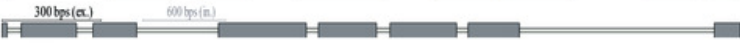

$\stackrel{3000 \mathrm{bs}}{\rightleftharpoons} \longrightarrow$
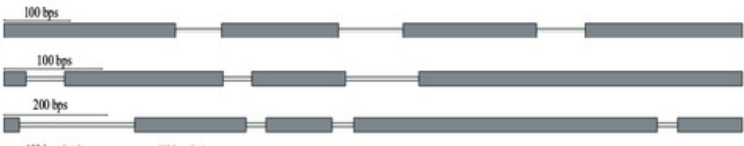

totots (xa)

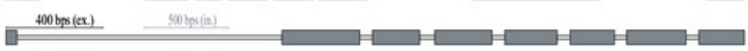

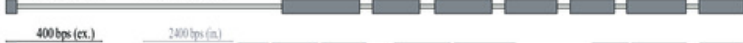

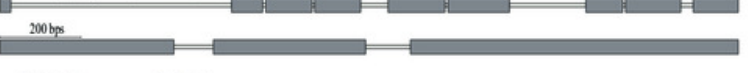

$300 \operatorname{tos}(2 x)$

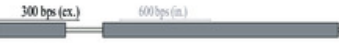

1 cobps
Subcelluar localization Mit Cyt Pox

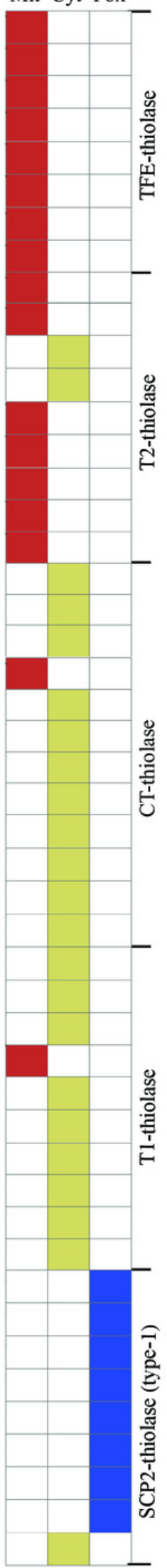




\section{Figure 4}

Fig. 4. Conserved domains and fingerprints of insect thiolases compared with humans and bacterium M. tuberculosis.

(A) The conserved domains predicted by hmmscan against Pfam database. Three types of domains were found in insect thiolases, including $\mathrm{N}$-terminal of thiolase (Thiolase_N), Cterminal of thiolase (Thiolase_C), and sterol carrier protein 2 (SCP2). BmorSCP2 (type-1) and BmorT1-1 were used to represent the common structures, respectively. (B) Highly conserved sequence blocks containing fingerprints $\mathrm{CxS}, \mathrm{NEAF}, \mathrm{GHP}$, and CxGGGxG. Three catalytic residues responsible for thiolase activity are indicated by stars. The alignement logos were generated by WebLogo (http://weblogo.berkeley.edu/logo.cgi). 


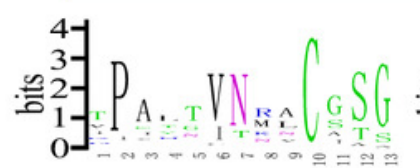

Lep_BmorSCP2 (type-1) Hym_AmelSCP2 (type-1) Dip_DmelSCP2 (type-1)-1 Dip_DmelSCP2 (type-1)-2 Col_TcasSCP2 (type-1) Lep_PxylSCP2 (type-1) HsapSCP2 (type-1) MtubSCP2 (type-1) MtubSCP2 (type-2) Lep_BmorT1-1 Lep_BmorT1-2 Lep_BmorT1-3 Hym_AmelT1

Dip_DmelT1

Col_TcasT1

Lep_PxylT1-1

Lep_PxylT1-2

Lep_PxylT1-3

Lep_PxylT1-4

Lep_PxylT1-5

Lep_PxylT1-6

Lep_PxylT1-7

Lep_PxylT1-8

Lep_PxylT1-9

Lep_PxylT1-10

HsapT1

Lep_BmorT2

Hym_AmelT2

Dip_DmelT2

Col_TcasT2

Lep_PxylT2

HsapT2

MtubT2

Hym_AmelCT

Dip_DmelCT

Col_TcasCT

Lep_PxylCT

HsapCT

HsapAB

Lep_BmorTFE

Hym_AmelTFE

Dip_DmelTFE

Col_TcasTFE

Lep_PxylTFE-2

Lep_PxylTFE-1

HsapTFE

MtubTFE

MtubTFEL (type-2)

MtubTFEL (type-1)-1

MtubTFEL (type-1)-3

MtubTFEL (type-1)-2

MtubTLP
73 I P I YNV NN NCS T G 77 CP I Y N V N N NCS T G 76 I PVY N V N N N C S T G 76 I PV Y N V N N N C S T G 189 I P I F N V N N N C S T G 73 I P I Y N V N NCS T G 85 I P I I N V N N N C A T G 92 I P I V N V N N NCS T G 84 TPATRHEA A C A S G 85 KPV L G I N R L C G S G 87 KPAL G V N R LCGS G 85 R PAL GVSKACG T G 85 KPS F S LNR LCGS G 85 KPAL G I N R L C G S G 85 R P A F L V NR L C G S G 38 KPV L G V NR L C G S G 341 T P A L G V N R L C G S G 84 RPAL V V NMAC GAG 84 R P A L AV N L A C G A G 84 RP ALG V N L AC GAG 84 RPALAV NLACGAG

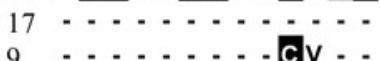
85 K PV L GV NR L C $\begin{array}{ll}85 & \text { KPV L GVNR LCGS } \\ 43 & \text { HPR . . . . . E S }\end{array}$ 83 T PALL T I N R LCG S G 66 T I C T T V N K V C A S G 66 T I C T T V N K V C A S G 100 V C C T T V NK V C S S G 101 T I C T T I N K V C A S G $111 \mathrm{~T}$ I C T T I N K V C S S G 117 T PC T T I N K V C A S G 75 V PAL T I N K M C L S G 81 T PAY L I NM L C G S G 79 V PAY G I NM L C G S G 80 V PAY T I NM L C G S G 79 V SA I T I NDVC GS G 83 VPAWS C QM I C GS G 114 V PLS T V NR QCS S G 141 T P A H T V T M AC I S S 117 I P A H T V T M AC I S S 119 T P A H T V T M AC I S S 124 T P A H T V T M A C I S S 123 T P A H T V T M A C I S A 47 TPA P T V T MAC I SA 129 T P A H T V T M A C I S A 90 TPAF D LQACG T G 81 LPG T T V NR Y C S S S 83 S G G V Q LNR F CAS G 80 VPG V T VDRQCGSS 84 V GA T T VDC QC G S G 91 MPQS LVNRACL - $\overline{\mathrm{CxS}}$

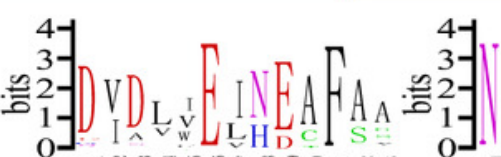

$V \iiint_{D} I_{L} A_{S}$

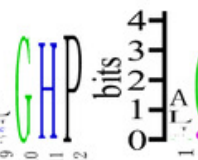

287 Q V DV V EL H D C F AA 290 D V DV I EL H D C F S I 290 D V Q V V EL H D C F S A 290 D V Q V V EL H D C F S A 402 D V Q V V EL H D C F S A 287 Q I DV V EL H D C F A A 298 D I DV I EL H D C F S T 303 DF G V I EL H D C F S A 300 DLDG I EV H D C F P S 313 D VD L I E I N EA F V A 317 D I D L V E I N EAFAA 315 D V D L FE I N EV F A S 315 D I EL I E I N EA F G S 315 D I D L I E I N EA F AA 315 D I D L I E I NEA F A A 266 D VD L I EVR - VY - 571 D V D L V E I N EA F AA 307 D V D L I E I N ETTF AA 308 G V D L I E I N ET TF A A 308 G V D L I E I N E T F A A 307 S V D L I E I N ET T A A 192 D V D L V E I N EA F A A 76 D V D L VE I N EA F A A 313 D V D L I E I N EA F C A 144 D V D L I E I N EA F C A 313 DMD L V EVN E A F AP 295 D V A L WE I N EA F S V 292 D I A L WE I N EA F S V 329 D V A MWEVN EA F S L 331 D V A LWE I N EA F S V 341 EV A LWELN EA F S V 346 D I A MWEV N EA F S L 306 Q L DV V E I N EA F AA 313 EVD L Y ELIN EA F A A 308 E V D L Y EL N EA F A A 306 D V D L Y EL N E A F A V 290 D V D L I EV N EA F A A 314 D V D I FE I N EA F A A 338 D V D I FE I NEAFAS 385 D I D T WE I HEA F A G 361 D I G V WE I HEA F A G 363 D I DSWE I HEA F A G 368 D I A V WEF HEA F A G 367 D I D T WE I HEA F A G 291 D I D T WE I HEA F A G 372 D I D A F EF HEA F S G 334 DF DF Y E I HEA F A S 321 D I D L V E I N EA F AV 320 D I D L FELN E A F A S 303 D I D T V E I N EA F A P 308 D I D I VE I NEAFAS 312 D I EY VDLY S CF S A NEAF
334 N P S G GL I AK G H P 337 N P S G GL I S K G H P 337 N P S G GL I S K G H P 337 NP S G GL I S K GH P 449 N P S G GL I S K G H P 334 N P S G GL I A K G H P 345 N P S G GL I S K G H P 350 N P S G GL I S K G H P 347 N P S G GL I - - GH P 342 N V N G G A T A L G H P 346 N V N G G A I A M G H P 344 N V N G G A L A F G N P 344 N V D G G S I A L G H P 344 N V N G G A I A L G H P 344 N V D G G A I A L G H P 288 - - G GQL S V - H P 600 N V S G G A I A M G H P 336 N V N G G A LA V G H P 337 N V N G G A LA V G H P 337 N V N G G A LA V G H P 336 N V N G G A L A V G H P 221 N V N G G A I A M G H P 105 N V N G G A I A M G H P 342 N V N G G A T A L G H P 173 N V N G G A T A L G H P 342 N V N G G A I A L G H P 324 N V H G G A V S L G H P 321 N I HG GAV S L G H P 358 N V H G G AV S I G H P 360 N V H G G AV S L G H P 370 N V H G G A V S I G H P 375 N I N G G AV S L G H P 335 N V N G G A I AV G H P 342 N I N G G A I A L G H P 337 N V N G G A I A L GH P 335 N I T G GA I A L GH P 319 N V N G G A I A L G H P 343 N I E G G A I A L G H P 367 N P L G G AV A L G H P 431 N K WG GS L S I G H P 407 N A WG G S L S I G H P 409 N N WG GS L S I G H P 414 N N WG G S L S I G H P 413 N N W G G S L S I G H P 337 N N WG GS L S I GH P 418 N N WG G S L S L G H P 380 N V N GS S LAA G H P 350 N I S G G A I A L G H P 349 N V N G G A I A M G H P 332 N P N G G A I A L G H P 337 N V N G GA I A L GH P 345 T V T G G - L T F G GP GHP
377 L Q L G G GMG 380 L Q I G G GLG 380 L Q L G G G L G 380 L Q L G G GL G 492 L Q I G G G L G 377 L Q L G G G M G 388 L Q L G G G L G 393 L Q I G G G - A 386 A - E A G G - G 381 A C I G G GQG 385 A C I G G GQG 383 S C - G G GQG 384 A C I G G G Q G 383 A C I G G GQ G 383 A C I G G GQ G 329 V C D G G GLG 639 A C I G G GQG 375 S C - G G GQG 384 S C - G G GQG 376 S C-G G GQ 365 -

260 A C I G G GQ G 144 A C I G G GQG 381 A C I G G GMG 202

381 A C I G G GQ 361 I C N G G G - G 358 I C N G G G - G 395 I C N G G G - G 397 I C N G G G - G 407 I C N G G G - G 412 I C N G G G - G 374 L C - G G GQG 381 L C I G G GM G 376 L C I G G G M G 374 L C I G G G M G 358 L C I G G G Q G 382 L C I G G GM G 408 M C I G G-M G 470 A C A A G G Q G 446 A C A A G G Q G 448 A C A A G G Q G 453 A C A A G G Q G 452 A C A A G G Q G 376 A C A A G GQG 457 A C A A G G Q G 426 I CA A G GQG 389 M C V G G G Q G 388 L C I G G G M G 371 M C EG G G T A 376 M C A G GA L G 384 A - N G G GV G

CxGGGxG 


\section{Figure 5}

Fig. 5. Protein homology modeling and 3D structures of insect thiolases.

The monomer structures of some representative thiolases were shown, including BmorT2 (A), DmelCT (B), PxutAB-1 (C), BmorT1-1 (D), BmorTFE (E), and BmorSCP2 (type-1) (F). The corresponding templates for homology modeling were PDB ID 6bjb.1.A, 4wyr.1.A, 1afw.1.A, 4wyr.1.A, 6dv2.1.A, and 6hrv.2.A, respectively. The dotted surface of the three catalytic residues was presented in (A) to (F) monomer structures. (G) Homo-tetramer of BmorT1-1. (H) Homo-dimer of BmorSCP2 (type-1). 
(A) $\mathrm{T} 2$

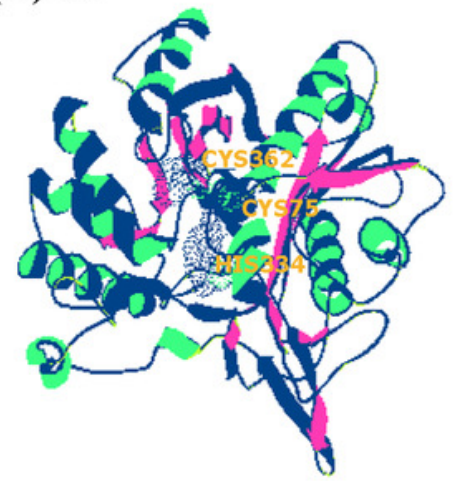

(D) $\mathrm{T} 1$

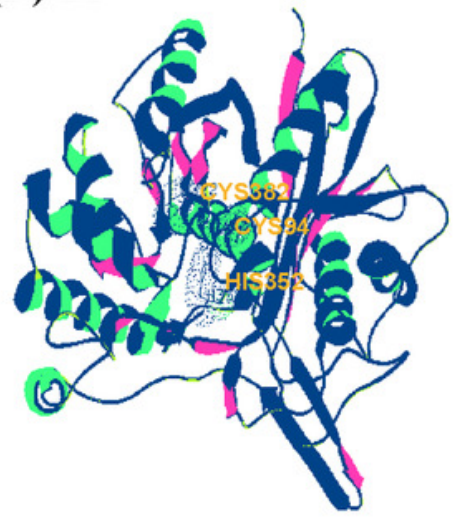

(G) Homo-tetramer of T1

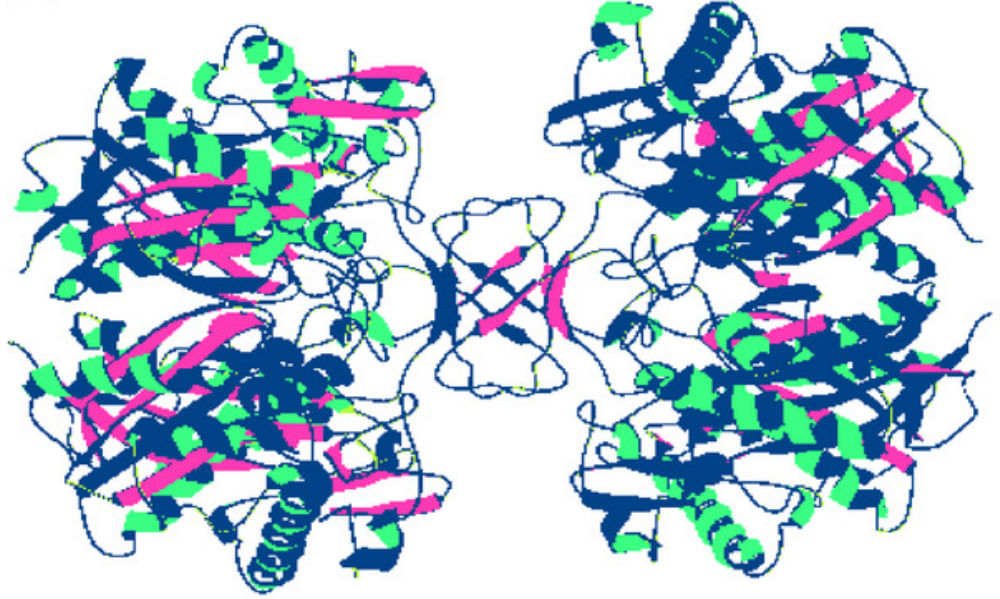

(B) CT

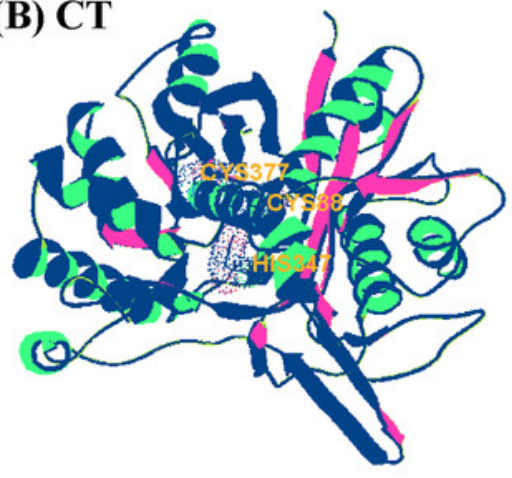

(E) TFE

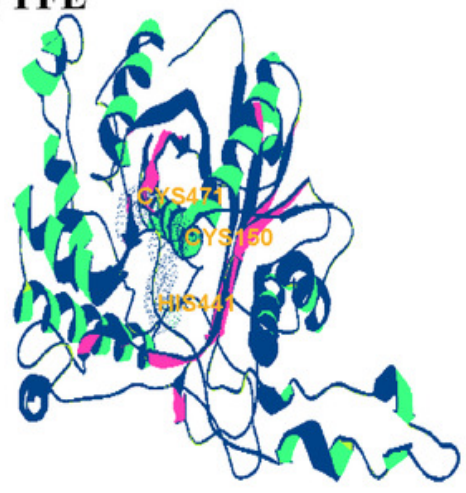

(C) AB

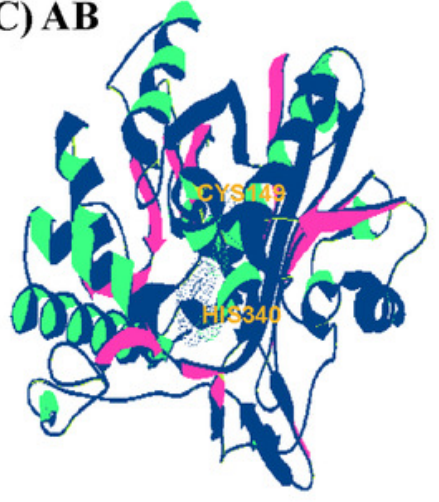

(F) SCP2 (type-1)

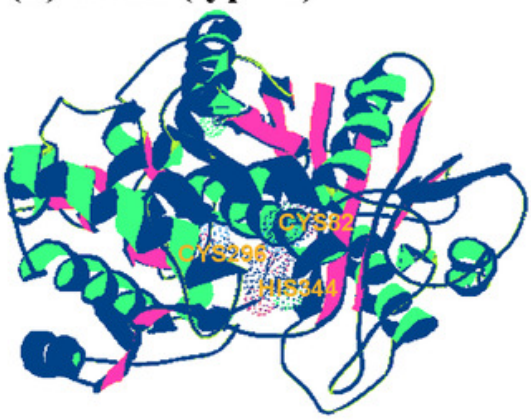

(H) Homo-dimer of SCP2 (type-1)

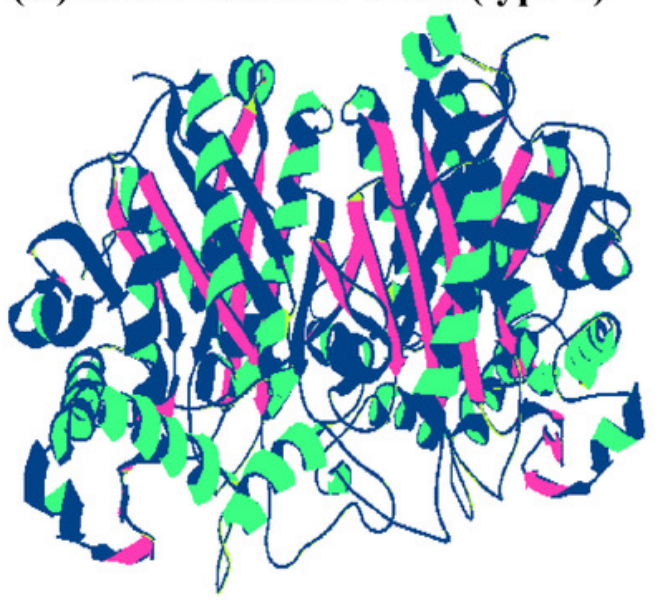




\section{Figure 6}

Fig. 6. Expression profiles of the thiolase genes in the silkworm.

(A) A chromatic scale diagram of expression levels in various tissues on day 3 of the fifth-instar larvae. The expression signal values were from the silkworm microarray, and signal values $<400$ were recognized no expression (Xia et al. 2007). (B) Expression patterns of the thiolase genes in the various tissues of the fifthinstar larvae. Expression signal of BmorT1-3 was not detected. It was not presented in the figure. (C) Expression profiles in the sex pheromone glands at different developmental stages of females. Expressions of BmorT1-2 and BmorT1-3 were not detectable in the PGs. 0-h and 24-h vergin: 0-h and 24-h old vergin adults after eclosion ; 3-h, 6-h and 9-h mated: female moths mated 3 hours, 6 hours and 9 hours.

(A)

$$
\begin{array}{r}
\text { BmorSCP2-sw01406 } \\
\text { BmorT2-sw21913 } \\
\text { BmorT1-1-sw12385 } \\
\text { BmorT1-3-sw21057 }
\end{array}
$$

BmorTFE-sw07055
Male on day 3 of fifth-instar larvae

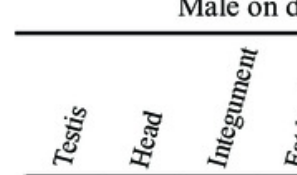

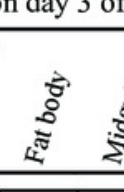

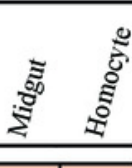

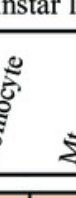

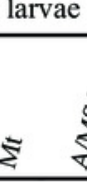

窟

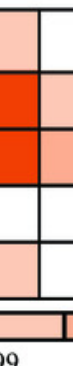

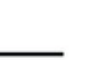

要

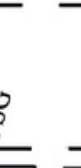

Female on day 3 of fifth-instar larvae

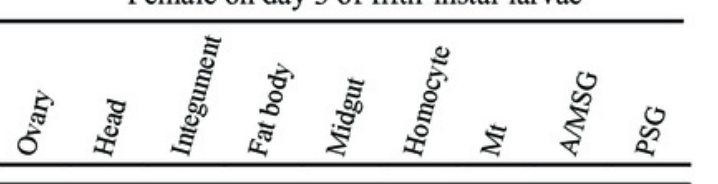

Expression signal value $<400.00$

400.00-1999.99

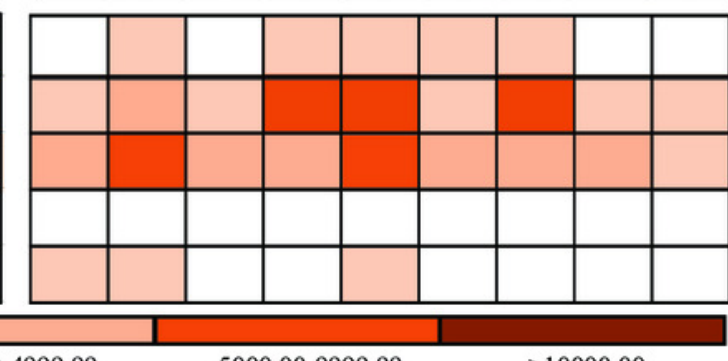

2000.00-4999.99

5000.00-9999.99

$>10000.00$

(B)

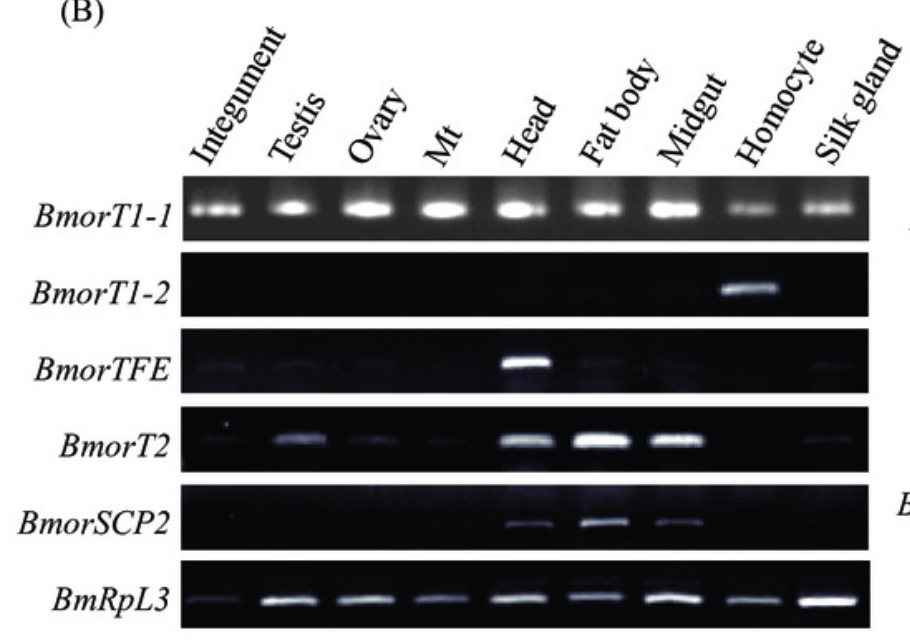

(C)

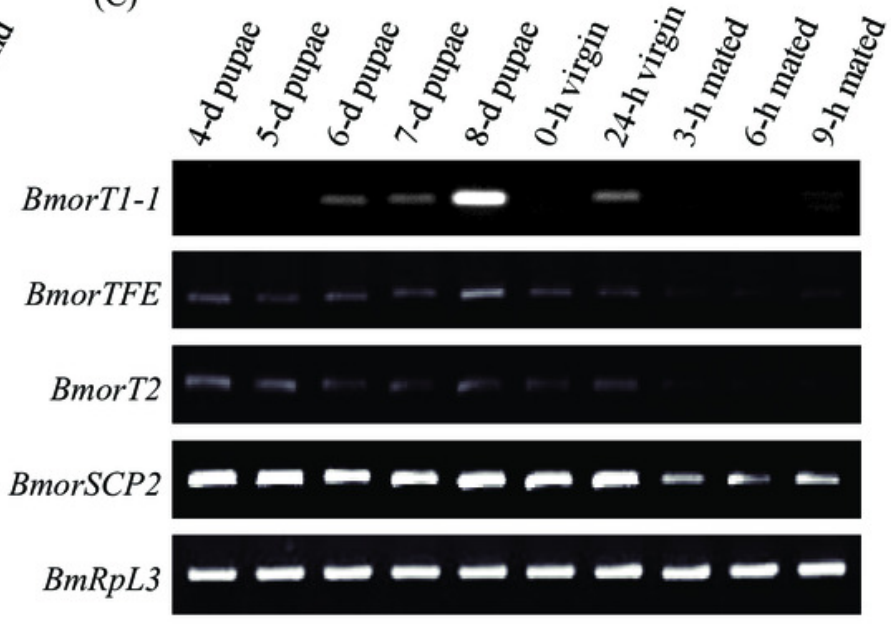




\section{Table $\mathbf{1}$ (on next page)}

Table 1. Classification of the thiolase genes among insects, $H$. sapiens and $M$. tuberculosis.

Lep, Lepidoptera; Dip, Diptera; Col, Coleoptera; Hem, Hemipter; Hym, Hymenoptera; Pht, Phthiraptera; Iso, Isoptera. Thiolase-like protein (TLP) of M. tuberculosis was not listed in the table, and was not found homologous genes in insects and human. 
Table 1 Classification of the thiolase genes among insects, H. sapiens and M. tuberculosis

\begin{tabular}{|c|c|c|c|c|c|c|c|c|c|c|}
\hline Organisms & Total & $\mathrm{CT}$ & $\mathrm{T} 1$ & $\mathrm{~T} 2$ & $\mathrm{AB}$ & TFE & $\begin{array}{c}\text { TFEL } \\
\text { (type-1) }\end{array}$ & $\begin{array}{c}\text { TFEL } \\
\text { (type-2) }\end{array}$ & $\begin{array}{c}\text { SCP2 } \\
\text { (type-1) }\end{array}$ & $\begin{array}{c}\mathrm{SCP} 2 \\
\text { (type-2) }\end{array}$ \\
\hline H. sapiens & 6 & 1 & 1 & 1 & 1 & 1 & & & 1 & \\
\hline M. tuberculosis & 9 & & & 1 & & 1 & 3 & 1 & 1 & 1 \\
\hline Lep_B. mori & 6 & & 3 & 1 & & 1 & & & 1 & \\
\hline Lep_P.xuthus & 10 & & 2 & 2 & 2 & 1 & & & 3 & \\
\hline Lep_M. sexta & 6 & & 3 & 1 & & 1 & & & 1 & \\
\hline Lep_D. plexippus & 5 & & 2 & 1 & & 1 & & & 1 & \\
\hline Lep_H. melpomene & 4 & & 1 & 1 & & 1 & & & 1 & \\
\hline Lep_P.xylostella & 15 & 1 & 10 & 1 & & 2 & & & 1 & \\
\hline Dip_C.quinquefasciatus & 6 & 2 & 1 & 1 & & 1 & & & 1 & \\
\hline Dip_A. gambiae & 7 & 1 & 2 & 1 & & 2 & & & 1 & \\
\hline Dip_D. melanogaster & 6 & 1 & 1 & 1 & & 1 & & & 2 & \\
\hline Col_A. glabripennis & 5 & & 2 & 2 & & & & & 1 & \\
\hline Col_N. vespilloides & 6 & 1 & 1 & 2 & & 1 & & & 1 & \\
\hline Col_T. castaneum & 5 & 1 & 1 & 1 & & 1 & & & 1 & \\
\hline Hem_H. halys & 5 & 1 & 1 & 1 & & 1 & & & 1 & \\
\hline Hem_A. pisum & 12 & 7 & 1 & 1 & & 1 & & & 2 & \\
\hline Hem_C. lectularius & 8 & 2 & 1 & 1 & & 2 & & 1 & 1 & \\
\hline Hym_D. alloeum & 8 & 2 & 1 & 3 & & 1 & & & 1 & \\
\hline Hym_A. mellifera & 5 & 1 & 1 & 1 & & 1 & & & 1 & \\
\hline Hym_B. impatiens & 8 & 1 & 2 & 2 & & 1 & & & 2 & \\
\hline Pht_P. humanus & 6 & 1 & 1 & 2 & & 1 & & & 1 & \\
\hline Iso_Z. nevadensis & 4 & 1 & 1 & 1 & & 1 & & & & \\
\hline
\end{tabular}

Nates. Lep, Lepidoptera; Dip, Diptera; Col, Coleoptera; Hem, Hemipter; Hym, Hymenoptera; Pht, Phthiraptera; Iso, Isoptera. Thiolase-like protein (TLP) of M. tuberculosis was not listed in the table, and was not found homologous genes in insects and hułnan. 